\title{
Quantum Mechanical (Phase Shift) Analysis of Differential Elastic Scattering of Molecular Beams*
}

\author{
RICHARD B. BERNSTEIN \\ Department of Chemistry, University of Michigan, Ann Arbor, Michigan
}

(Received March 9, 1960)

For a spherically symmetrical intermolecular potential $V(r)=$ $\epsilon f(r / \sigma)$ the quantum calculation of the elastic scattering cross section $d \sigma(\theta) / d \Omega$ in the c.m. system is carried out as follows. For a given relative velocity (or deBroglie wavelength) and an assumed $V(r)$, the radial wave equation is integrated for successive values of the angular momentum quantum number $l$, yielding the phase shifts $\eta_{l}$. Then $d \sigma(\theta) d \Omega$ is computed in terms of the series of $\eta_{i}$ 's in the standard way. A general computational program (following that of $\mathrm{K}$. Smith) is outlined for the evaluation of the radial wave function and the phase shifts, utilizing an IBM 704 computer. Calculations are presented for the L-J
$(12,6)$ potential function. The results may be concisely represented using the framework provided by the semiclassical treatment of Ford and Wheeler, i.e., in terms of a set of reduced phase constants vs reduced angular momenta at various reduced relative kinetic energies $K$. Tables and graphs are presented from which the phases may be obtained, to a good approximation, for any given $\epsilon, \sigma$ and $K$. Computation of the differential and total cross sections from the phase shifts is then readily accomplished.

The results are compared with the classical and semiclassical treatments. The problem of tunneling and orbiting is discussed.

\section{INTRODUCTION}

$\mathbf{F}$ OR molecules interacting according to a spherically symmetrical potential

$$
V(r)=\epsilon f(r / \sigma),
$$

the quantum mechanical problem of the elastic scattering of molecular beams is already solved, in principle, by the standard Mott-Massey theory. ${ }^{1}$ For a given relative velocity $v$ (or deBroglie wavelength $\lambda$ ) and an assumed $V(r)$ the radial wave function is integrated for successive values of the angular momentum quantum number $l$, yielding the phase constants $\eta_{l}$. Then the differential elastic scattering cross sections (in the c.m. system) $d \sigma(\theta) / d \Omega$ are computed in terms of the $\eta$ 's by the standard equation ${ }^{1}$

$$
\begin{aligned}
\frac{d \sigma(\theta)}{d \Omega} \equiv I(\theta) & =\frac{1}{4 k^{2}}\left\{\left[\sum_{l}(2 l+1) \sin 2 \eta_{l} P_{l}(\cos \theta)\right]^{2}\right. \\
& \left.+\left[\sum_{l}(2 l+1)\left(\cos 2 \eta_{l}-1\right) P_{l}(\cos \theta)\right]^{2}\right\},
\end{aligned}
$$

where $k=2 \pi / \lambda=\mu v / \hbar, \mu$ is the reduced mass, and $v$ is the initial relative speed; $P_{l}$ is the Legendre function.

In connection with the problem of the elastic scattering of electrons, Kenineth $\mathrm{Smith}^{2}$ has developed a computational program for evaluating the radial wave function from which the phase shifts may be calculated. This program has now been adapted to the molecular beam scattering problem, and extensive calculations have been carried out and are here reported for the commonly used Lennard-Jones $(12,6)$ potential func-

* The author acknowledges with thanks financial support of this work from the United States Atomic Energy Commission, Division of Research, and from the Alfred P. Sloan Foundation.

1 N. F. Mott and H. S. W. Massey, The Theory of Atomic Collisions (Clarendon Press, Oxford, England, 1949), 2nd ed.

2 Kenneth Smith, private communication, June 24, 1959. The computational scheme is outlined briefly in a report by $\mathrm{K}$. Smith, W. F. Miller, and A. J. Mumford, Argonne Natl. Lab., February 9,1960 tion, which may be expressed by

$$
V^{*}(x)=f(x)=4\left(x^{-12}-x^{-6}\right),
$$

where $x=r / \sigma$ and $V^{*}=V / \epsilon$ is the reduced potential.

\section{METHOD}

The differential equation for the radial wave function $R(r)$ is usually written ${ }^{1,3}$ in terms of $G(r)=r R(r)$ as

$d^{2} G_{l}(r) / d r^{2}+\left[k^{2}-U(r)-l(l+1) / r^{2}\right] G_{l}(r)=0$,

where

$k(=\mu v / \hbar)=\left(2 \mu E / \hbar^{2}\right)^{\frac{1}{3}}, \quad U(\boldsymbol{r})=\left(2 \mu / \hbar^{2}\right) V(\boldsymbol{r})$,

and $E=\frac{1}{2} \mu v^{2}$ is the initial relative kinetic energy.

The following dimensionless parameters are introduced:

$$
A=k \sigma \quad \text { and } \quad B=\left(2 \mu / \hbar^{2}\right) \epsilon \sigma^{2} .
$$

Eq. (4) becomes

$$
d^{2} y / d x^{2}+\left[A^{2}-B f(x)-l(l+1) / x^{2}\right] y=0,
$$

where $y(x) \equiv G_{l}(r)$. Alternatively, Eqs. (4) and (7) may be written in terms of the reduced relative kinetic energy, defined as

$$
K=E / \epsilon=A^{2} / B \text {. }
$$

Here $B$ may be considered an independent variable, with either $A$ or $K$ as the second independent variable. Then

$$
d^{2} y / d x^{2}+\left[1-f(x) / K-\beta^{2} / x^{2}\right] A^{2} y=0,
$$

where a reduced angular momentum function

$$
\beta=[l(l+1)]^{\frac{1}{2}} / A
$$

has been introduced. The "reduced effective potential"

${ }^{3}$ See, for example, L. I. Schiff, Quantum Mechanics (McGrawHill Book Company, Inc., New York, 1955), 2nd ed. 
TABLE I. $(A=20 ; B=125$. $)$

\begin{tabular}{ccccc}
\hline \hline$l$ & $x_{s}$ & $\Delta x$ & $y_{0}{ }^{\prime}$ & $\eta_{\text {calc }}$ \\
\hline 0 & 0.70 & 0.005 & 1 & -16.933 \\
& 0.65 & 0.005 & $10^{-30}$ & -16.933 \\
& 0.60 & 0.005 & $10^{-30}$ & -16.935 \\
& 0.60 & 0.020 & $10^{-30}$ & -16.947 \\
\multirow{2}{*}{1} & 0.70 & 0.005 & 1 & -15.420 \\
& 0.70 & 0.010 & 1 & -15.419 \\
& 0.60 & 0.010 & $10^{-30}$ & -15.421 \\
20 & 0.65 & 0.005 & $10^{-30}$ & +2.219 \\
& 0.60 & 0.005 & $10^{-30}$ & +2.219 \\
\hline \hline
\end{tabular}

$V_{\text {eff }} *$ may be defined by the relation

$$
\begin{aligned}
Z=V_{\mathrm{eff}}{ }^{*}=\left[V(r)+\frac{l(l+1)}{r^{2}} \frac{\hbar^{2}}{2 \mu}\right] / \epsilon & \\
& =f(x)+\frac{l(l+1)}{B x^{2}} .
\end{aligned}
$$

Thus Eq. (7) may also be written

$$
d^{2} y / d x^{2}+(K-Z) B y=0 .
$$

For $r$ sufficiently large so that $|U(\boldsymbol{r})| \ll k^{2}$, the solutions of Eq. (4) are',3

$$
\begin{aligned}
G_{l}(r) \sim(\pi k r / 2)^{\frac{1}{3}}\left[\cos \eta_{l} J_{l+\frac{1}{3}}(k r)\right. \\
\left.+(-1)^{l} \sin \eta_{l} J_{-l-\frac{1}{2}}(k r)\right],
\end{aligned}
$$

where the $J$ 's refer to the usual half-odd-order Bessel functions.

In terms of the reduced parameters,

$$
y=c\left[\cos \eta_{l} j_{l}(A x)-\sin \eta_{l} n_{l}(A x)\right],
$$

where $j_{l}(z)$ and $n_{l}(z)$ are the spherical Bessel and spherical Neumann functions, respectively, and $c$ is a constant independent of $x$. The phase shifts $\eta_{l}$ are defined $^{1}$ in terms of the asymptotic solutions of Eq. (4) as $r \rightarrow \infty$ :

or

$$
G_{l}(r) \sim \sin \left[k r-(\pi l / 2)+\eta_{l}\right]
$$

$y \sim \sin \left[A x-(\pi l / 2)+\eta_{l}\right]=\sin \left[A\left(x+\eta_{l}{ }^{*}\right)-(\pi l / 2)\right]$,

where $\eta_{l}{ }^{*}=\eta_{l} / A$.

The $\eta_{l}$ 's may be found from a knowledge only of the zeros of $y$ at large $x$. From Eq. (14), if $x_{n}$ is the $n$th zero of $y$, then

$$
\eta_{l}=\arctan \left[j_{l}\left(A x_{n}\right) / n_{l}\left(A x_{n}\right)\right] .
$$

Thus, the procedure is as follows. Apparent phase shifts are calculated for successive zeros of $y$ and are compared; when the difference between successively determined $\eta_{l}$ values becomes negligible it may be considered that the condition $\left(|U(\boldsymbol{r})| \ll k^{2}\right)$ necessary for the validity of Eq. (14) has been attained and that $\eta_{l}$ has been evaluated.
The procedure outlined thus far (which is standard in the field of nuclear scattering) has also been employed by a number of workers ${ }^{4-7}$ interested in the low temperature properties (especially the second virial coefficient) of helium. In the present instance, the computations were facilitated by the use of an IBM 704 computer. The standard Runge-Kutta-Gill (RKG) method was employed for the numerical integration of the wave equation.

A few features of the present computational program may be noted. First, regarding the boundary conditions, it was advantageous to modify the assumed potential so that for $x<x_{s}, V=\infty$ (and thus $y=0$ ), while for $x \geq x_{s}, V=\epsilon f(x)$ as usual. For the L-J $(12,6)$ case, it was found that all zeros of $y$ (and thus the phase shifts) were substantially independent of $x_{8}$ for $x_{8} \leq 0.7$ (corresponding to $V^{*} \geq 255$ ). In addition, it was noted ${ }^{2}$ that any arbitrary value of the initial slope, $y_{0}^{\prime}$, could be used; the zeros of $y$ are independent of $y_{0}{ }^{\prime}$. Lastly, as regards the interval size $\Delta x$ in the RKG integration, it was found that for $\Delta x \leq 0.005$, the zeros and phases were essentially independent of $\Delta x$; in most cases even 0.01 was adequate. The integration was carried out to a sufficiently large $x$ (typically in the range from 5 to 15) such that the differences between successive apparent phases were consistently less than some

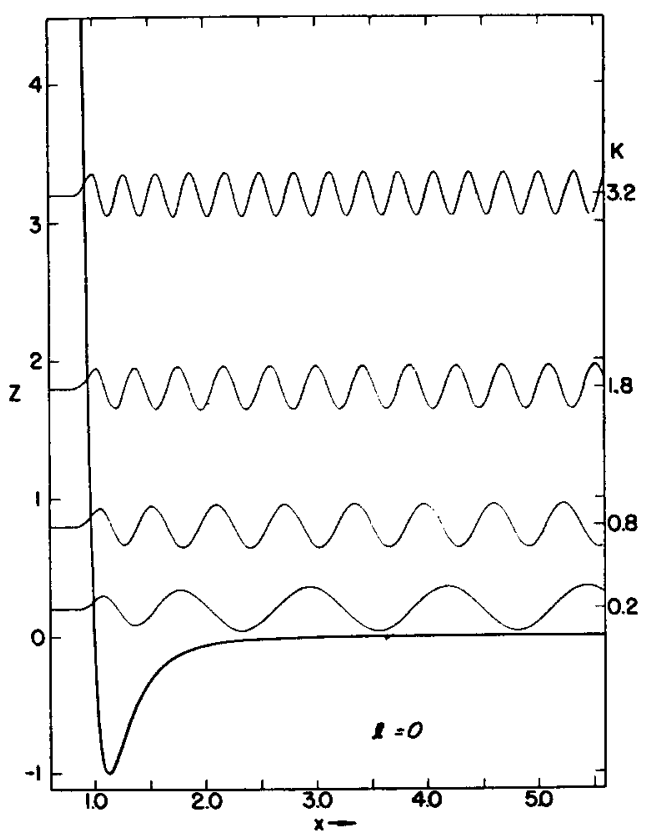

FIG. 1. Reduced effective potential and radial wave functions for $B=125$ and $l=0$.

${ }^{4}$ H. S. W. Massey and R. A. Buckingham, Proc. Roy. Soc. (London) A168, 378 (1938).

5 J. De Boer and A. Michels, Physica 6, 409 (1939).

(a) R. A. Buckingham, J. Hamilton, and H. S. W. Massey, Proc. Roy. Soc. (London) A179, 103 (1951); (b) R. A. Buckingham, A. R. Davies, and D. C. Gilles, Proc. Phys. Soc. (London)

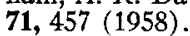

7 J. De Boer, Repts. Progr. Phys. 12, 351 (1949). 
predetermined value (usually $<0.0002 \mathrm{rad}$ ). The computation time was usually $\leq 1 \mathrm{~min}$.

Table I summarizes some of the results illustrating the independence of the calculated phase shift, $\eta_{\text {calc }}$, upon $x_{s}$ and $\Delta x$ for one of the least favorable cases (small ratio of $\lambda / \sigma$ ). On the basis of a number of check calculations made under varying conditions, it is estimated that the probable error in $\eta_{\text {calc }}$ is $\pm 0.002 \mathrm{rad}$.

The program for $d \sigma(\theta) / d \Omega$ calculated from the phases yielded values at $1^{\circ}$ intervals in $\theta$ from $0^{\circ}$ to $179^{\circ}$. This program was checked by a few direct calculations and by duplicating a number of calculations in the literature.

\section{RESULTS AND DISCUSSION}

The results of the present study may conveniently be presented in two parts. Part A summarizes a detailed investigation of the radial wave functions, phase shifts and scattering cross sections for one particular example, the parameters for which correspond to the $\mathrm{H}_{2}-\mathrm{Hg}$ system. The scattering of $\mathrm{H}_{2}$ beams by $\mathrm{Hg}$ has been measured by Knauer ${ }^{8}$ while classical cross section calculations are available ${ }^{9}$ for the $\mathrm{L}-J(12,6)$ potential. Part $B$ involves the transformation of these results into a form of more general applicability. The method employed takes advantage of certain of the features of the semiclassical treatment of Ford and Wheeler ${ }^{10}$ and consists of the evaluation of a set of reduced phase

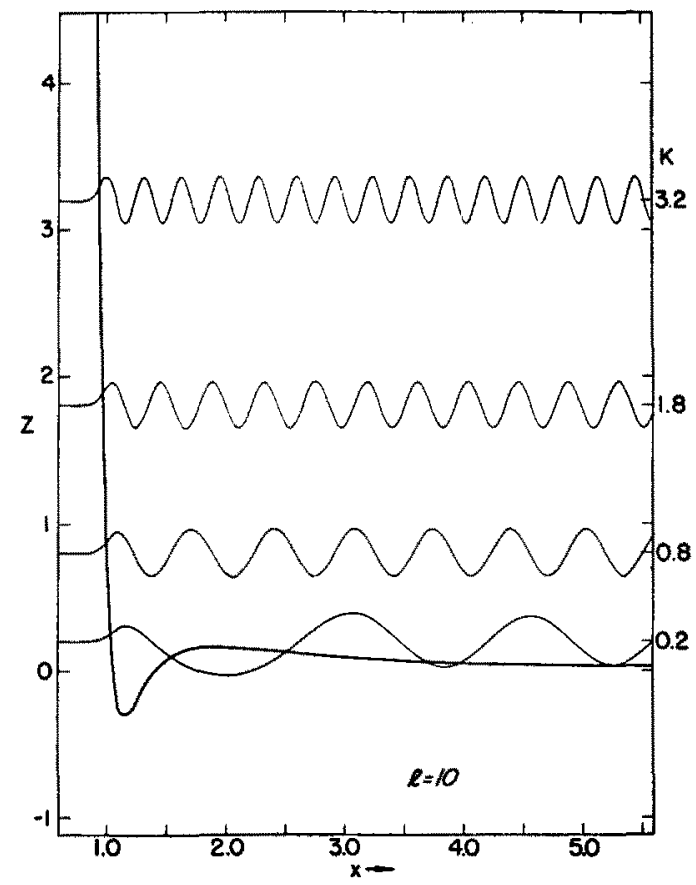

FrG. 2. Reduced effective potential and radial wave functions for $B=125$ and $l=10$.

${ }^{8}$ F: Knauer, Z. Physik 80, 80 (1933); 90, 559 (1934).

${ }^{2} H$. U. Hostettler and R. B. Bernstein, J. Chem. Phys. 31, $1422(1959)$.

10 K. W. Ford and J. A. Wheeler, Ann. Phys. 7, 259, 287 (1959).

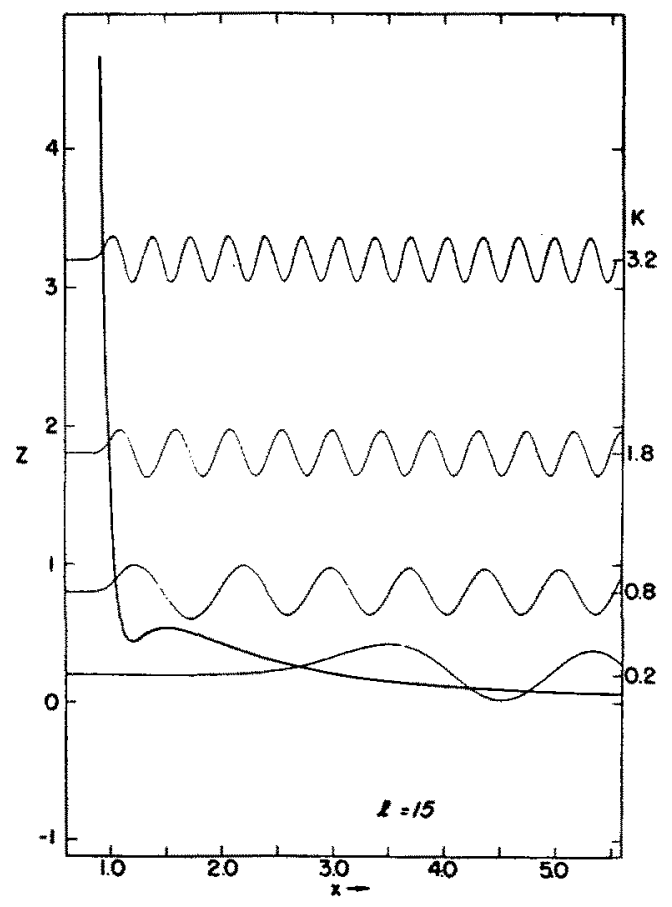

FrG. 3. Reduced effective potential and radial wave functions for $B=125$ and $l=15$.

constants vs reduced angular momenta at various reduced relative kinetic energies $K$. With the use of the tables and graphs presented, it is possible to construct a set of phase shifts $\eta_{l}$ for any L-J $(12,6)$ system of given $\epsilon, \sigma$ and $K$. Only in a certain region of $\eta-l-K$ space (where the mapping has not been sufficiently extensive) would it be necessary to perform any direct phase calculations; otherwise, the phases may be satisfactorily estimated by the use of the tables within $\pm 0.04 \mathrm{rad}$.

\section{A. Calculations for the System: $\mathbf{H}_{2}-\mathbf{H g}$}

The L-J $(12,6)$ parameters for the $\mathrm{H}_{2}-\mathrm{Hg}$ system are taken ${ }^{9}$ to be: $\epsilon=2.46 \times 10^{-14} \mathrm{erg}, \sigma=2.91 \times 10^{-8} \mathrm{~cm}$; thus, one obtains the value of $B=124.1$ [from Eq. (6)]. Figures 1-4 show plots of the reduced effective potential $Z$ vs $x$ for the rounded value $B=125$ and $l=0,10,15$, and 20 .

Although only the zeros of $y$ are needed for evaluation of the phases, the complete radial wave function was an optional byproduct of the phase calculation. A number of representative wave functions were thus computed, as listed in Table II. Plotted on Figs. 1-4 are a few of the calculated radial wave functions $y(x)$, all drawn with the same asymptotic $(x \rightarrow \infty)$ amplitude, and vertically placed to indicate the reduced relative kinetic energy $K$. Shown are wave functions for $K=0.2,0.8,1.8$, and 3.2 , which correspond to $A=5$, 10,15 , and 20 , respectively. The asymptotic $(x \rightarrow \infty)$ wavelengths are inversely proportional to $A$. The classical turning points (i.e., the distance of closest 


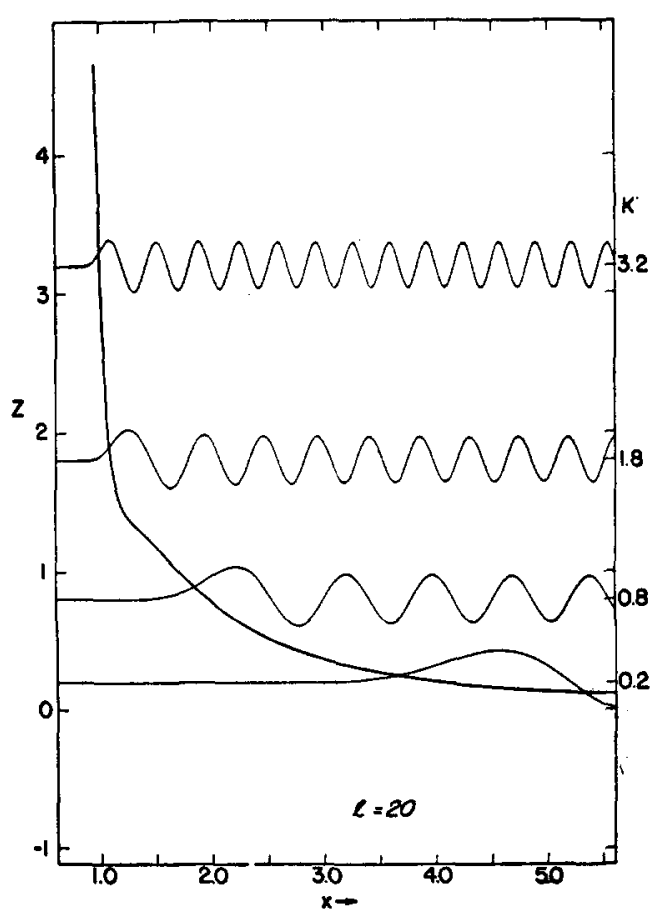

FIG. 4. Reduced effective potential and radial wave functions for $B=125$ and $l=20$.

approach) are located at the first inflection point in the wave function [from Eq. (12), $y^{\prime \prime}=0$ when $Z=K$ ]. The distortion of the wave function in the region of the attractive well and the influence of the centrifugal barrier are to be noted.

The wave functions reveal a number of interesting features, particularly with regard to the classical phenomena of orbiting ${ }^{11}$ and rainbow scattering, ${ }^{12}$ associated with the existence of the hump in the effective potential curve. Hirschfelder et al. ${ }^{11}$ have shown classically that for the L-J $(12,6)$ potential, orbiting will occur for any $K \leq 0.8$ at some particular value (dependent on $K$ ) of the reduced angular momentum, if $\beta^{2} K \leq 2.4624$. In terms of the classical deflection function $\Theta(b)$ this corresponds to a certain impact parameter $b_{0}$ for which $\Theta\left(b_{0}\right) \rightarrow-\infty$. For $K>0.8$ the deflection function goes only to a finite

TABLE II. List of wave functions computed for $B=125$.

\begin{tabular}{rl}
\hline$A$ & \multicolumn{1}{r|}{$l$} \\
\hline 3 & $0,2,4,6,7,8,10$ \\
5 & $0,2,5,9,10,11,12,15,20,25,30$ \\
7 & $8,10,11,12,13,14,15,16,17,20$ \\
9 & $0,12,13,14$ \\
10 & $0,3,5,8,10,15,16,17,18,20$ \\
15 & $0,3,10,15,20$ \\
20 & $0,1,2,3,4,5,6,7,8,10,15,20,25,30,40,50$ \\
\hline
\end{tabular}

11 J. O. Hirschfelder, C. F. Curtiss, and R. B. Bird, Molecular Theory of Gases and Liquids (John Wiley \& Sons, Inc., New York, 1954), pp. 553-557.

12 See footnote reference 10, pp. 313-322. minimum, giving rise to rainbow scattering rather than orbiting. In the present notation and for $B=125$ the conditions for classical orbiting are therefore as follows: $A^{2} / B \leq 0.8$, or $A \leq 10$, and $l$ less than some value $l_{0}$ given approximately by $l_{0} \cong(2.4624 B)^{2} \cong 17.5$ \{obtained using Eq. (10), approximating $\left[l_{0}\left(l_{0}+1\right)\right]^{\frac{1}{2}}$ by $\left.l_{0}\right\}$.

As pointed out by Ford and Wheeler, ${ }^{12}$ penetration of the centrifugal barrier (tunneling) should occur for $K$ slightly less than $Z$ in the region of the hump. Wave functions illustrating this effect are shown in Fig. 5. Plotted are the curves for $K=0.20 \quad(A=5)$ with

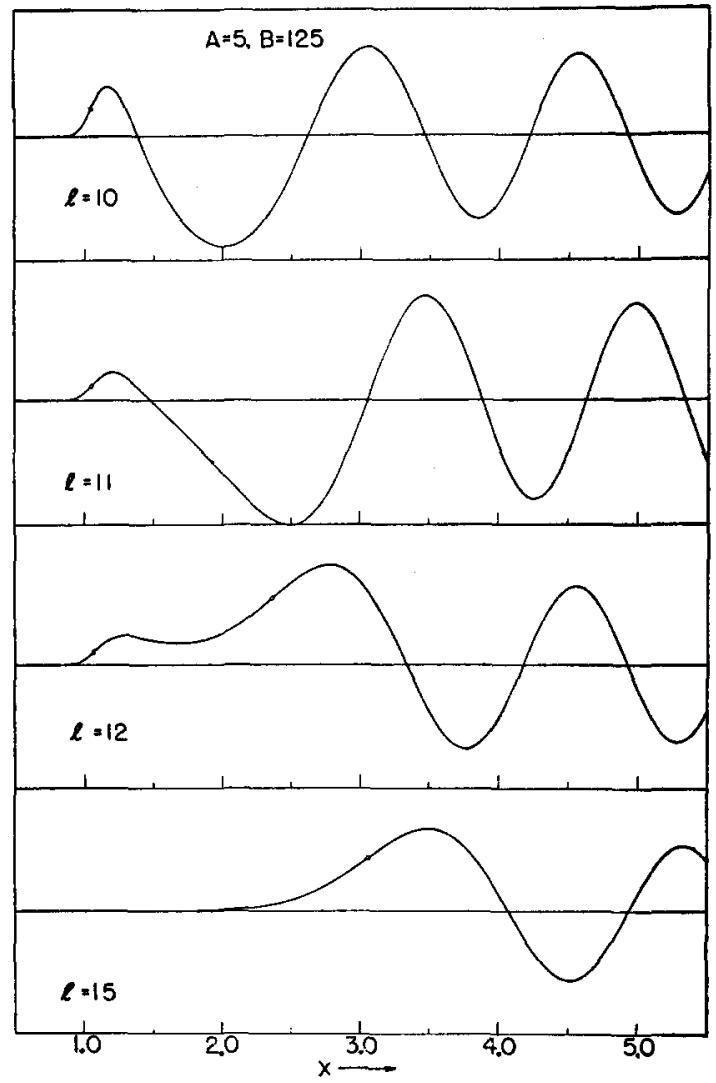

FIG. 5. Radial wave functions illustrating phenomenon of tunneling through the centrifugal barrier.

$l=10,11,12$, and 15 . (It should be pointed out that, in contrast to Figs. 1-4, the amplitudes here are arbitrary and unrelated.) The barrier penetration is apparent in the curve for $l=12$. One also notes the "loss" of a zero in going from $l=11 \rightarrow 12$; this gives rise to an abrupt change in phase shift (as anticipated from footnote reference 12). Similar results were obtained in other cases where $A \leq 10$ and $l \leq 17$. Of interest also is the location of the (classical) turning points, designated on the curves by small circles; the $x$ on the curve for $l=12$ is, of course, classically inaccessible.

Table III is a summary of the phase shifts (in radians) for the present example $(B=125)$. The values quoted with three decimal places are computed phase 
Table IIL. Phase shifts $(\eta l)$ for $B=125$.

\begin{tabular}{|c|c|c|c|c|c|c|c|c|c|c|c|}
\hline$\stackrel{A}{A}$ & 3 & 5 & 7 & 9 & 10 & $\lambda^{A}$ & 15 & 18 & 20 & 24 & 30 \\
\hline $\begin{array}{r}0 \\
1 \\
2 \\
3 \\
4 \\
5 \\
6 \\
7 \\
8 \\
9 \\
10 \\
11 \\
12 \\
13 \\
14 \\
15 \\
16 \\
17 \\
18 \\
19 \\
20 \\
21 \\
22 \\
23 \\
24 \\
25 \\
26 \\
27 \\
28 \\
29 \\
30 \\
31 \\
32 \\
33 \\
34 \\
35 \\
36 \\
37 \\
38 \\
39 \\
40 \\
41 \\
42 \\
43 \\
44 \\
45\end{array}$ & $\begin{array}{c}+2.551 \\
-3.92 \\
4.994 \\
5.82 \\
6.427 \\
6.75 \\
6.653 \\
6.302 \\
3.603 \\
3.329 \\
3.246 \\
0.064 \\
0.041 \\
0.027 \\
0.019 \\
0.013\end{array}$ & $\begin{array}{l}-0.484 \\
+0.95 \\
2.179 \\
3.263 \\
4.175 \\
4.906 \\
5.440 \\
5.758 \\
5.826 \\
5.526 \\
4.557 \\
3.788 \\
0.406 \\
0.25 \\
0.157 \\
0.110 \\
0.079 \\
0.06 \\
0.05 \\
0.04 \\
0.027 \\
0.02 \\
0.02 \\
0.02 \\
0.01 \\
0.009 \\
0.01 \\
0.01 \\
0.01 \\
0.01 \\
0.004\end{array}$ & $\begin{array}{l}-3.140 \\
-1.69 \\
-0.371 \\
+0.84 \\
1.90 \\
2.814 \\
3.62 \\
4.25 \\
4.713 \\
5.01 \\
5.097 \\
4.928 \\
4.473 \\
3.817 \\
0.872 \\
0.490 \\
0.331 \\
0.236 \\
0.17 \\
0.13 \\
0.102 \\
0.08 \\
0.06 \\
0.05 \\
0.04 \\
0.033 \\
0.03 \\
0.02 \\
0.02 \\
0.02 \\
0.014\end{array}$ & $\begin{array}{l}-5.565 \\
-4.10 \\
-2.731 \\
-1.47 \\
-0.33 \\
+0.727 \\
1.69 \\
2.50 \\
3.168 \\
3.74 \\
4.152 \\
4.43 \\
4.509 \\
4.395 \\
4.043 \\
3.212 \\
1.371 \\
0.780 \\
0.533 \\
0.37 \\
0.292 \\
0.23 \\
0.18 \\
0.14 \\
0.11 \\
0.092 \\
0.08 \\
0.06 \\
0.05 \\
0.04 \\
0.037 \\
0.03 \\
0.03 \\
0.02 \\
0.02 \\
0.017\end{array}$ & $\begin{array}{l}-6.716 \\
-5.24 \\
-3.86 \\
-2.572 \\
-1.38 \\
-0.297 \\
+0.68 \\
1.56 \\
2.336 \\
3.02 \\
3.521 \\
3.90 \\
4.168 \\
4.254 \\
4.150 \\
3.805 \\
2.977 \\
1.550 \\
0.919 \\
0.64 \\
0.468 \\
0.35 \\
0.27 \\
0.21 \\
0.17 \\
0.142 \\
0.12 \\
0.10 \\
0.08 \\
0.07 \\
0.057 \\
0.05 \\
0.05 \\
0.04 \\
0.03 \\
0.026 \\
0.02 \\
0.02 \\
0.02 \\
0.02 \\
0.014 \\
0.01 \\
0.01 \\
0.01 \\
0.01 \\
0.008\end{array}$ & $\begin{array}{r}0 \\
1 \\
2 \\
3 \\
4 \\
5 \\
6 \\
7 \\
8 \\
9 \\
10 \\
11 \\
12 \\
13 \\
14 \\
15 \\
16 \\
17 \\
18 \\
19 \\
20 \\
21 \\
22 \\
23 \\
24 \\
25 \\
26 \\
27 \\
28 \\
29 \\
30 \\
31 \\
32 \\
33 \\
34 \\
35 \\
36 \\
37 \\
38 \\
39 \\
40 \\
41 \\
42 \\
43 \\
44 \\
45 \\
46 \\
47 \\
48 \\
49 \\
50\end{array}$ & $\begin{array}{l}-12.051 \\
-10.56 \\
-9.13 \\
-7.750 \\
-6.45 \\
-5.231 \\
-4.10 \\
-3.06 \\
-2.06 \\
-1.08 \\
-0.223 \\
+0.54 \\
1.22 \\
1.80 \\
2.31 \\
2.731 \\
3.03 \\
3.209 \\
3.260 \\
3.18 \\
2.880 \\
2.39 \\
1.836 \\
1.36 \\
1.04 \\
0.808 \\
0.64 \\
0.51 \\
0.42 \\
0.35 \\
0.298 \\
0.25 \\
0.21 \\
0.18 \\
0.16 \\
0.135 \\
0.12 \\
0.10 \\
0.09 \\
0.08 \\
0.069 \\
0.06 \\
0.05 \\
0.04 \\
0.04 \\
0.038 \\
0.03 \\
0.03 \\
0.03 \\
0.03 \\
0.023\end{array}$ & $\begin{array}{l}-15.022 \\
-13.52 \\
-12.08 \\
-10.69 \\
-9.35 \\
-8.060 \\
-6.84 \\
-5.70 \\
-4.63 \\
-3.61 \\
-2.640 \\
-1.76 \\
-0.97 \\
-0.24 \\
+0.45 \\
1.096 \\
1.63 \\
2.06 \\
2.400 \\
2.65 \\
2.800 \\
2.84 \\
2.750 \\
2.531 \\
2.196 \\
1.809 \\
1.453 \\
1.165 \\
0.944 \\
0.774 \\
0.642 \\
0.54 \\
0.46 \\
0.39 \\
0.33 \\
0.284 \\
0.24 \\
0.21 \\
0.18 \\
0.16 \\
0.144 \\
0.13 \\
0.11 \\
0.10 \\
0.09 \\
0.080 \\
0.07 \\
0.06 \\
0.05 \\
0.05 \\
0.047\end{array}$ & $\begin{array}{l}-16.933 \\
-15.420 \\
-13.964 \\
-12.547 \\
-11.197 \\
-9.898 \\
-8.662 \\
-7.471 \\
-6.351 \\
-5.278 \\
-4.266 \\
-3.32 \\
-2.431 \\
-1.61 \\
-0.846 \\
-0.156 \\
-0.472 \\
1.03 \\
1.503 \\
1.89 \\
2.219 \\
2.46 \\
2.576 \\
2.607 \\
2.531 \\
2.344 \\
2.08 \\
1.768 \\
1.469 \\
1.213 \\
1.008 \\
0.840 \\
0.707 \\
0.600 \\
0.51 \\
0.432 \\
0.37 \\
0.32 \\
0.28 \\
0.25 \\
0.219 \\
0.20 \\
0.17 \\
0.15 \\
0.14 \\
0.12 \\
0.11 \\
0.10 \\
0.08 \\
0.08 \\
0.070\end{array}$ & $\begin{array}{l}-20.634 \\
-19.10 \\
-17.639 \\
-16.22 \\
-14.84 \\
-13.483 \\
-12.17 \\
-10.93 \\
-9.767 \\
-8.63 \\
-7.535 \\
-6.50 \\
-5.504 \\
-4.55 \\
-3.67 \\
-2.851 \\
-2.10 \\
-1.40 \\
-0.75 \\
-0.11 \\
+0.440 \\
0.92 \\
1.32 \\
1.656 \\
1.92 \\
2.105 \\
2.23 \\
2.26 \\
2.184 \\
2.05 \\
1.874 \\
1.66 \\
1.447 \\
1.25 \\
1.07 \\
0.925 \\
0.81 \\
0.70 \\
0.61 \\
0.53 \\
0.464 \\
0.41 \\
0.36 \\
0.32 \\
0.28 \\
0.255 \\
0.23 \\
0.21 \\
0.19 \\
0.17 \\
0.150\end{array}$ & $\begin{array}{c}-25.941 \\
-24.38 \\
-22.85 \\
-21.41 \\
-20.00 \\
-18.60 \\
-17.29 \\
-16.02 \\
-14.78 \\
-13.56 \\
-12.392 \\
-11.24 \\
-10.16 \\
-9.09 \\
-8.08 \\
-7.10 \\
-6.20 \\
-5.32 \\
-4.50 \\
-3.70 \\
-2.981 \\
-2.30 \\
-1.65 \\
-1.08 \\
-0.55 \\
-0.04 \\
+0.41 \\
0.800 \\
1.12 \\
1.38 \\
1.592 \\
1.736 \\
1.819 \\
1.843 \\
1.81 \\
1.739 \\
1.64 \\
1.51 \\
1.37 \\
1.23 \\
1.094 \\
0.97 \\
0.86 \\
0.77 \\
0.69 \\
0.62 \\
0.55 \\
0.50 \\
0.46 \\
0.42 \\
0.368\end{array}$ \\
\hline
\end{tabular}

shifts (with a probable error of \pm 0.002 , as mentioned previously), while those given to two decimal places are interpolated values, with a pe of \pm 0.02 .

Figures 6 and 7 show graphically the dependence of the phase shifts upon $l$ at different values of $A$. The dotted lines are used simply to connect the different parts of the curves, i.e., to cross the discontinuities which arise from the "loss" of a zero. Figures $8(a)$ and 8(b) show the dependence of $\eta_{l}$ upon $A$.

As generally understood, the short-range repulsive part of the potential is responsible for the negative loworder phases, while the long-range attractive part gives rise to the positive higher-order phases. As the relative kinetic energy is decreased the influence of the attrac- tive well becomes dominant; conversely, as the collision energy increases the negative phases predominate. The low-order phases tend to approach those ${ }^{13}$ for the rigid-sphere case (where, for example, $\eta_{0}=-A$ ). Inspection of Table III also shows that the value of $l$ corresponding to a maximum in $\eta_{l}$ is a linear function of $A$ (i.e., in the present example, for $A>3, l_{\max }=A+3$ ).

For the higher collision energies, as the semiclassical approximation becomes more valid, the initial slopes $(d \eta / d l)_{0}$ are found to approach the expected value of

${ }^{13}$ H. S. W. Massey and C. B. O. Mohr, Proc. Roy. Soc. (London) A141, 434 (1933). 


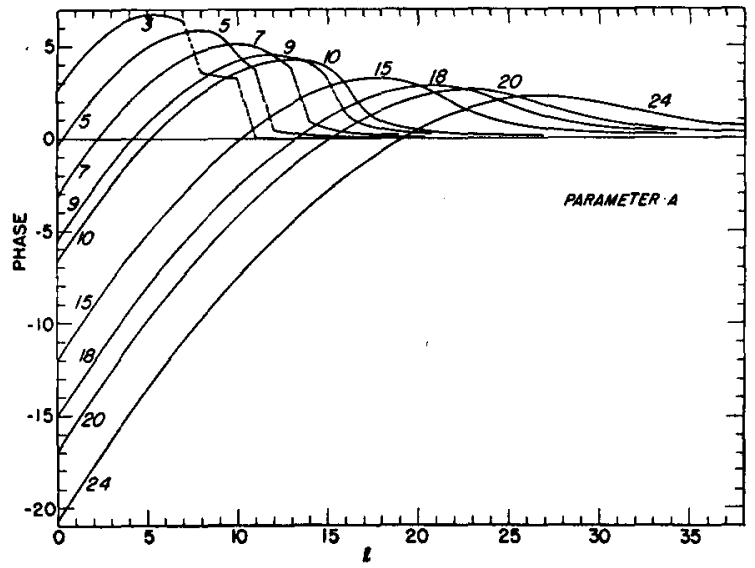

Fig. 6. Phase constant vs angular momentum quantum number.

$\pi / 2$. Using the semiclassical correspondence relation,

$$
\Theta(l)=2(d \eta / d l),
$$

for the deflection function, $\Theta(l)$ was calculated for several values of $A$; the curves were quite similar to those calculated ${ }^{9}$ classically, differing primarily in the region near the minimum in $\Theta$.

A comparison of quantum vs classical calculation of the scattering cross sections is shown in Fig. 9. Plotted is the angular dependence of the scattering calculated [from Eq. (2)] for the case of $A=14.1$ ( $K=1.6)$ using phases interpolated from Figs. 6-8, compared with the classically calculated ${ }^{9}$ curve for the same $K$. The predicted ${ }^{14}$ discontinuity at the characteristic angle $\chi_{s}$ (designated "rainbow scattering" in footnote reference 10) is not evident in the quantum calculation.

In Fig. $10 \mathrm{Knauer}^{8} \mathrm{~s}^{8}$ experimental data are compared with the quantum calculation for $A=18(K=2.6)$, which corresponds fairly closely with the "most probable" value of $A$ under the conditions of the experiments. Properly, one should average over the

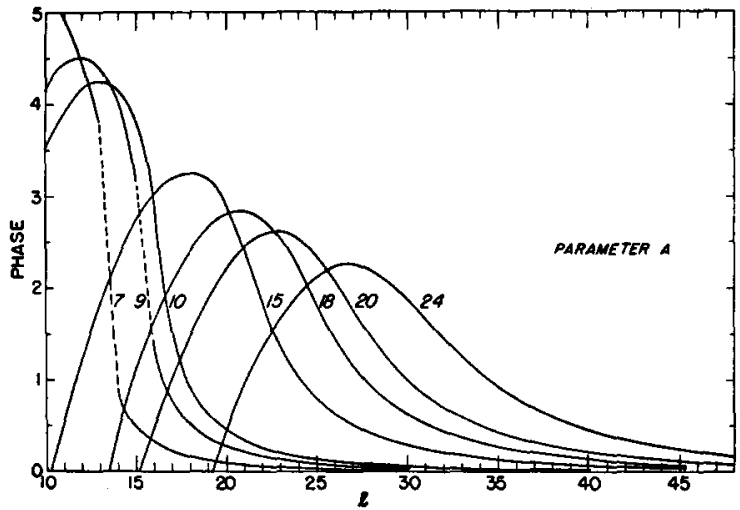

FIG. 7. Phase constant vs angular momentum quantum number.

${ }_{14}$ E. A. Mason, J. Chem. Phys. 26, 667 (1957). appropriate distribution of relative velocities, which would, of course, destroy most or all of the undulating character of the quantum curve, and give more nearly the observed monotonic dependence of $d \sigma / d \Omega$ upon $\theta$. In addition, phenomena connected with rotation of the $\mathrm{H}_{2}$ molecule and the spin of the protons will tend to wash out the structure that would be expected for the scattering of one ideal, monoenergetic, $J=0$ system by another.

\section{B. Generalization in Terms of Reduced Parameters}

In the Ford-Wheeler ${ }^{12}$ semiclassical treatment the

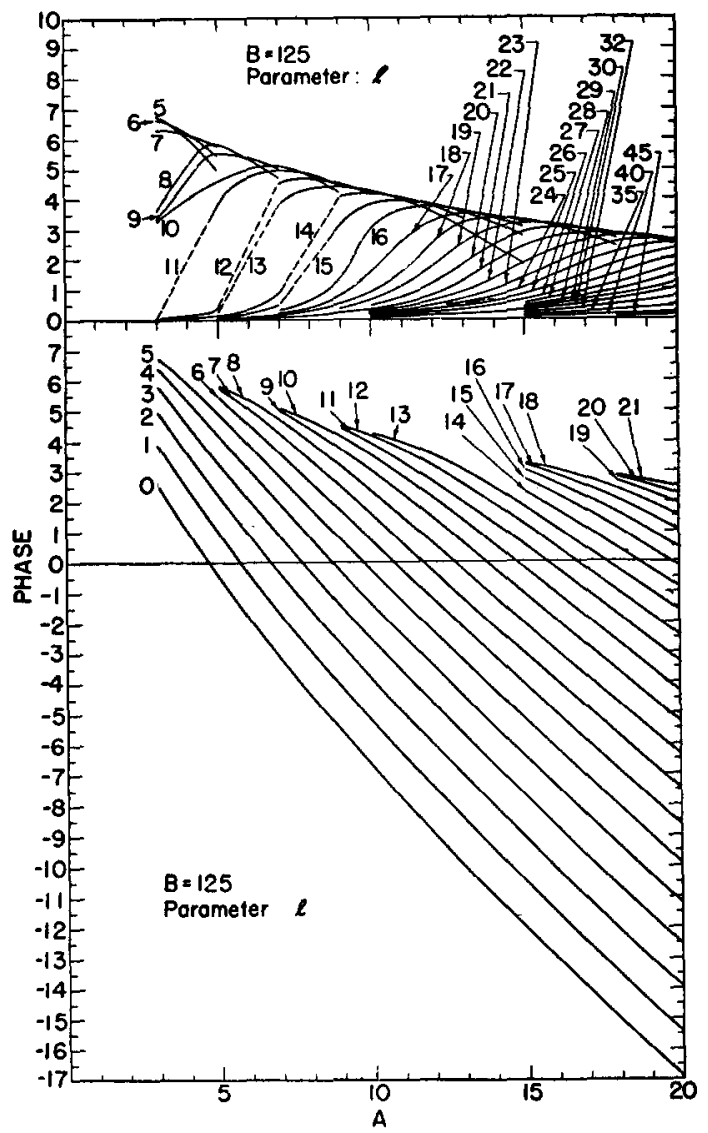

(a)

(b)

FIg. 8. Phase constant vs velocity parameter, $A$.

reduced phase constant is defined as

$$
\eta^{*}=\eta /(\mu \nu \sigma / \hbar)=\eta / A \text {. }
$$

This is the same as the $\eta^{*}$ used in Eq. (16).

For fixed $v$, the deflection function is given ${ }^{12}$ by

$$
\Theta=2\left(d \eta^{*} / d b^{*}\right),
$$

where $b^{*}$ is the reduced impact parameter, ${ }^{11} b^{*}=b / \sigma$. The reduced centrifugal potential analogous to the term $l(l+1) / x^{2} B$ in Eq. (11) is $K b^{* 2} / x^{2}$, so that the reduced angular momentum is $K b^{* 2}$. The previously defined [Eq. (10)] reduced angular momentum function $\beta$ is then identified with $b^{*}$. 
According to the semiclassical treatment, a set of curves of $\eta^{*}$ vs $b^{*}$ for various values of $K$ should be unique (i.e., independent of the deBroglie wavelength or of $\epsilon)$. In footnote reference 10 (p. 317) two such curves (for $K=0.4$ and 1.6) together with the corresponding deflection functions are shown.

Referring back to the quantum treatment, it is not obvious from Eq. (9) that, for a given $K$ and $\beta$, a unique reduced phase constant $\eta^{*}[\mathrm{Eq}$. (16)] would be obtained independent of $A$ (or $B$ ). In this connection, it is interesting to note the implication of the semiclassical treatment, i.e., that specification of $B$ and $K$ suffices to determine $\eta^{*}\left(b^{*}\right)$ and thus $\Theta\left(b^{*}\right)$. In the quantum treatment, Eq. (9) suggests that $\eta^{*}(\beta)$ might require the specification of $A$ as well as $B$ and $K$, i.e., a wavelength dependence of the reduced phase constants (which would presumably disappear with increasing $A$ ).

Figure 11 shows the results of the present calculations (for $B=125$ ) plotted as $\eta^{*}$ vs $\beta$ for various $K$ 's. The dotted curves identify the abscissa $\beta$ as distinct from $\beta^{\prime}$ (defined later) wherever the two are different. These $\eta^{*}$ vs $\beta$ curves are unsatisfactory for two reasons. First, the semiclassical constraint on initial slopes,

$$
\left(d \eta^{*} / d b^{*}\right)_{0}=\pi / 2
$$

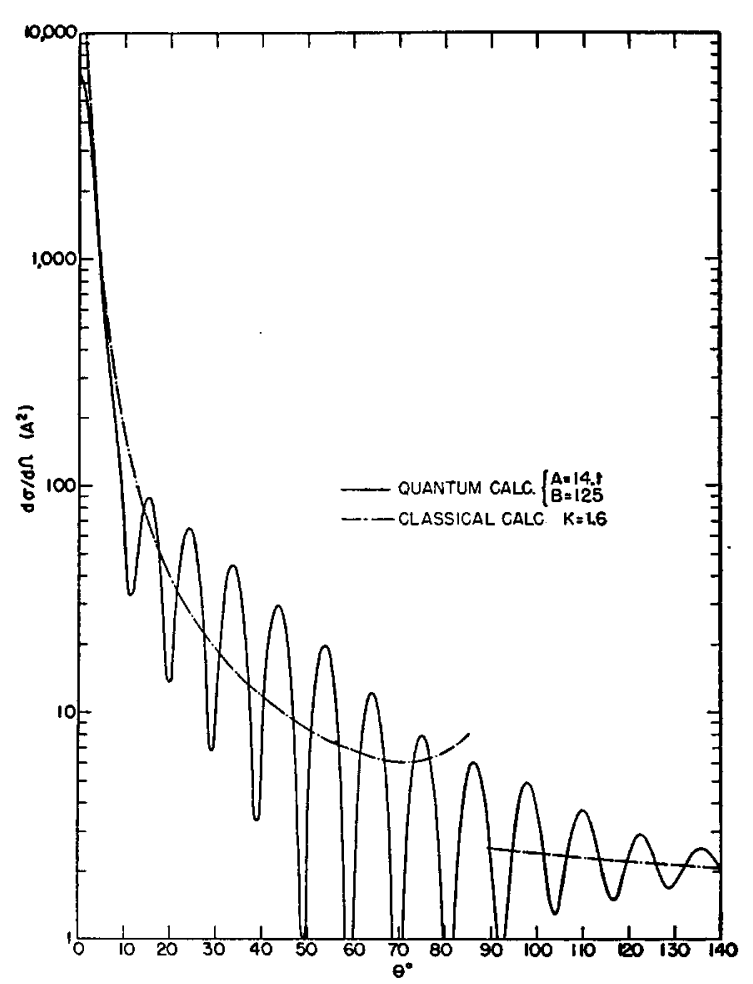

Fig. 9. Classical vs quantum calculation of the scattering of $\mathrm{H}_{2}$ by $\mathrm{Hg}$ in the idealization where both systems are considered to have zero angular momentum. In actuality the undulations will be partly washed out by effects associated with the rotation of the $\mathrm{H}_{2}$ molecule, the spin of the protons, and the difficulty of securing monoenergetic beams.

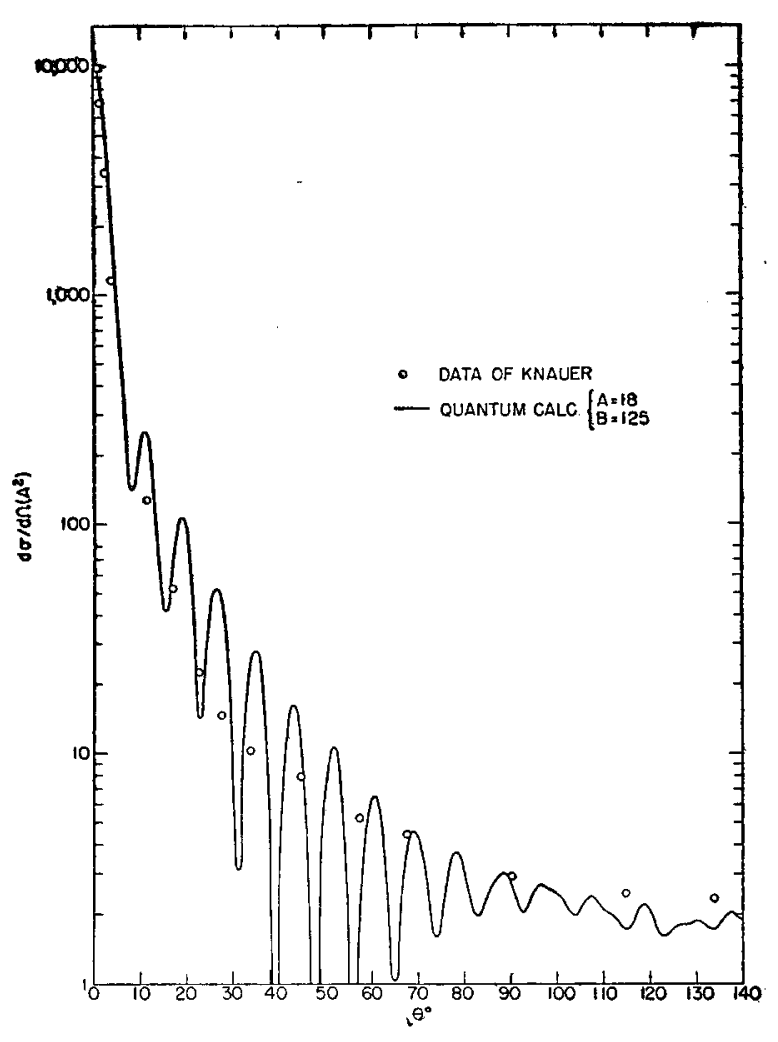

Fig. 10. Scattering of $\mathrm{H}_{2}$ by $\mathrm{Hg}$ at $295^{\circ} \mathrm{K}$. (See the remark on the quantum calculations in the caption of Fig. 9.)

is not satisfied (in fact, an inflection point is noted at small $\beta$ ); second, calculations of $\eta_{l}$ made with different $B$ and the same $K$ yielded points $\left(\beta, \eta^{*}\right)$ which were not on the same iso- $K$ curve.

It is well known $n^{1,10,15,16}$ that increased accuracy is obtained in the JWKB approximation for the phases by replacing (in the centrifugal potential term) the quantity $l(l+1)$ by $\left(l+\frac{1}{2}\right)^{2}$ (the so-called Langer modification). ${ }^{16}$ In order to take advantage of as much as possible of the framework of the semiclassical treatment, a modified reduced angular momentum function was defined as

$$
\beta^{\prime}=\left(l+\frac{1}{2}\right) / A .
$$

Of course, for large $l, \beta^{\prime} \cong \beta \cong l / A$, (as implied in footnote reference 10, p. 318 ).

The solid curves in Fig. 11 refer to abscissa $\beta^{\prime}$. These curves are found to satisfy the condition of Eq. (21). Moreover, at low $\beta^{\prime}$ they agree closely with the iso-K curves of $\eta^{*}$ vs $b^{*}$ plotted in footnote reference $10, \mathrm{p}$. 317. (However, for $b^{*}$ greater than that corresponding to the maximum in $\eta^{*}$, the differences become appreciable.)

The important question, however, is not the extent of the agreement with the semiclassically estimated

\footnotetext{
${ }^{15}$ K. W. Ford, D. L. Hill, M. Wakano and J. A. Wheeler, Ann. Phys. 7, 239 (1959)
}

${ }_{16}$ R. E. Langer, Phys. Rev. 51, 669 (1937). 


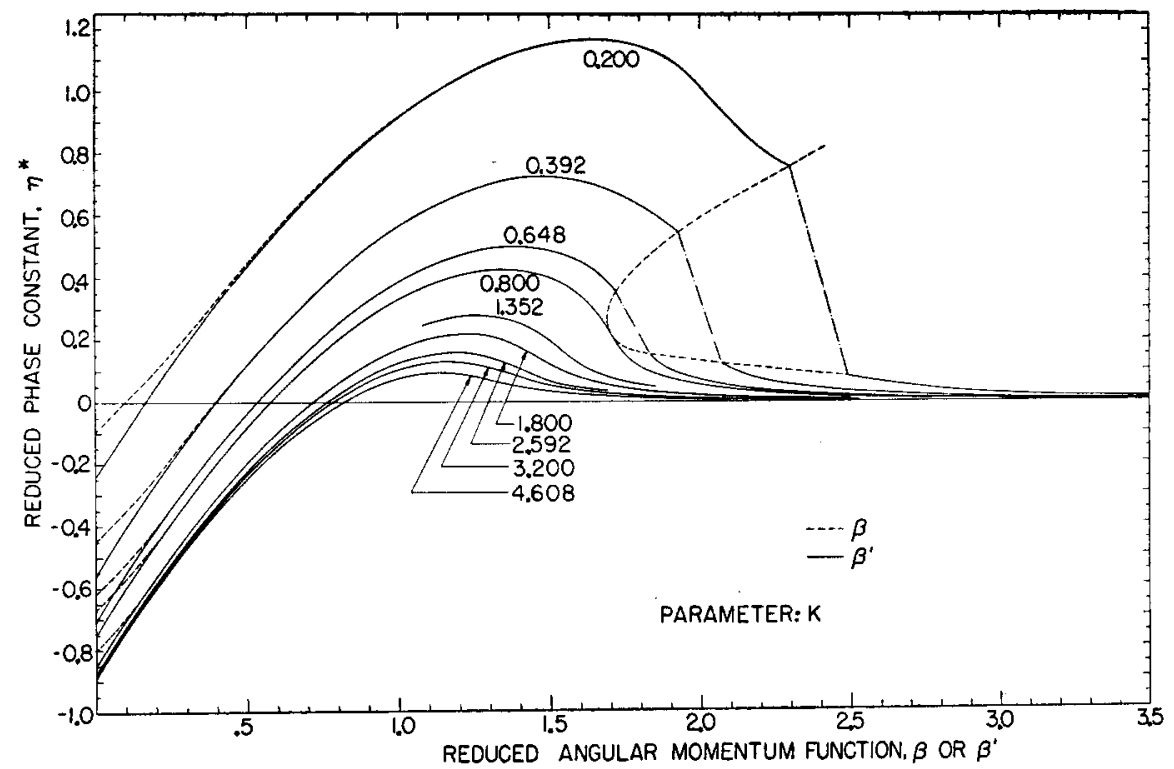

Fig. 11. Reduced phase constant vs reduced angular momentum functions.

TABle IV. Estimated vs calculated phase shifts.

\begin{tabular}{|c|c|c|c|c|c|c|c|c|}
\hline$K$ & $B$ & $A$ & $l$ & $\eta_{\text {cale }}{ }^{*}$ & $\eta_{\text {est }}{ }^{*}$ & $\eta_{\text {OAlo }}$ & $\eta_{\text {est }}$ & $\eta_{\text {est }}-\eta_{\text {ealc }}$ \\
\hline 0.200 & 375 & 8.66 & $\begin{array}{r}0 \\
2 \\
5 \\
10 \\
20 \\
30\end{array}$ & $\begin{array}{r}-0.155 \\
+0.176 \\
+0.591 \\
+1.052 \\
+0.108 \\
+0.011\end{array}$ & $\begin{array}{r}-0.157 \\
+0.174 \\
+0.586 \\
+1.046 \\
+0.106 \\
+0.012\end{array}$ & $\begin{array}{r}-1.34 \\
+1.52 \\
+5.12 \\
+9.11 \\
+0.93 \\
+0.10\end{array}$ & $\begin{array}{r}-1.36 \\
+1.51 \\
+5.08 \\
+9.05 \\
+0.92 \\
+0.10\end{array}$ & $\begin{array}{r}-0.02 \\
-0.01 \\
-0.04 \\
-0.06 \\
-0.01 \\
0.00\end{array}$ \\
\hline 0.267 & 375 & 10.00 & $\begin{array}{r}0 \\
5 \\
10 \\
20 \\
30\end{array}$ & $\begin{array}{l}-0.319 \\
+0.342 \\
+0.783 \\
+0.743 \\
+0.017\end{array}$ & $\begin{array}{r}-0.322 \\
+0.339 \\
+0.777 \\
+0.744 \\
+0.016\end{array}$ & $\begin{array}{r}-3.19 \\
+3.42 \\
+7.83 \\
+7.43 \\
+0.17\end{array}$ & $\begin{array}{r}-3.22 \\
+3.39 \\
+7.77 \\
+7.44 \\
+0.16\end{array}$ & $\begin{array}{l}-0.03 \\
-0.03 \\
-0.06 \\
+0.01 \\
-0.01\end{array}$ \\
\hline 0.800 & 250 & 14.14 & $\begin{array}{r}0 \\
2 \\
3 \\
5 \\
10 \\
20 \\
30\end{array}$ & $\begin{array}{r}-0.693 \\
-0.485 \\
-0.388 \\
-0.208 \\
+0.152 \\
+0.417 \\
+0.035\end{array}$ & $\begin{array}{l}-0.695 \\
-0.486 \\
-0.390 \\
-0.210 \\
+0.152 \\
+0.415 \\
+0.035\end{array}$ & $\begin{array}{r}-9.79 \\
-6.86 \\
-5.48 \\
-2.95 \\
+2.15 \\
+5.90 \\
+0.49\end{array}$ & $\begin{array}{l}-9.82 \\
-6.87 \\
-5.51 \\
-2.97 \\
+2.15 \\
+5.87 \\
+0.49\end{array}$ & $\begin{array}{r}-0.03 \\
-0.01 \\
-0.03 \\
-0.02 \\
0.00 \\
-0.03 \\
0.00\end{array}$ \\
\hline 1.200 & 62.5 & 8.66 & 0 & -0.727 & -0.728 & -6.29 & -6.30 & -0.01 \\
\hline 1.600 & 62.5 & 10.00 & 0 & -0.770 & -0.770 & -7.70 & -7.70 & 0.00 \\
\hline 1.800 & 62.5 & 10.61 & $\begin{array}{r}0 \\
2 \\
5 \\
10 \\
20 \\
30\end{array}$ & $\begin{array}{r}-0.784 \\
-0.515 \\
-0.183 \\
+0.163 \\
+0.026 \\
+0.003\end{array}$ & $\begin{array}{l}-0.781 \\
-0.515 \\
-0.182 \\
+0.164 \\
+0.025 \\
+0.003\end{array}$ & $\begin{array}{l}-8.31 \\
-5.47 \\
-1.94 \\
+1.73 \\
+0.28 \\
+0.04\end{array}$ & $\begin{array}{l}-8.28 \\
-5.47 \\
-1.93 \\
+1.74 \\
+0.27 \\
+0.04\end{array}$ & $\begin{array}{r}+0.03 \\
0.00 \\
+0.01 \\
+0.01 \\
-0.01 \\
0.00\end{array}$ \\
\hline 3.200 & 62.5 & 14.14 & $\begin{array}{r}0 \\
2 \\
5 \\
10 \\
20 \\
30\end{array}$ & $\begin{array}{r}-0.832 \\
-0.626 \\
-0.359 \\
-0.031 \\
+0.067 \\
+0.008\end{array}$ & $\begin{array}{l}-0.835 \\
-0.625 \\
-0.357 \\
-0.029 \\
+0.066 \\
+0.008\end{array}$ & $\begin{array}{r}-11.76 \\
-8.85 \\
-5.07 \\
-0.44 \\
+0.94 \\
+0.11\end{array}$ & $\begin{array}{r}-11.80 \\
-8.84 \\
-5.05 \\
-0.41 \\
+0.93 \\
+0.11\end{array}$ & $\begin{array}{r}-0.04 \\
+0.01 \\
+0.02 \\
+0.03 \\
-0.01 \\
0.00\end{array}$ \\
\hline
\end{tabular}

curves, but rather whether the iso- $K$ curves obtained from the quantum calculations (the " $Q-i-K$ " curves) are unique (i.e., independent of $B$, for example). To investigate this point, a number of phase shift calculations were carried out with different $B$ (and $A$ ); the range of $B$ was from 62.5 to 375 , with the range of $K$ from 0.2 to 3.2. The results are presented in Table IV. The $\eta_{\text {calc }}{ }^{*}$ values refer to the directly calculated reduced phases, while the $\eta_{\mathrm{est}}{ }^{*}$ values are taken from the data of Fig. 11 (with abscissa $\beta^{\prime}$ ). The correspond- 
ing phase shifts $\eta$ are also listed. The average deviation $\eta_{\text {est }}-\eta_{\text {ealc }}$ is $\pm 0.02 \mathrm{rad}$. Thus it appears that the present $Q-i-K$ curves are very nearly unique, at least over the range of practical interest. Without a rigorous theoretical foundation, however, this result must be regarded as semi-empirical.

Figures 12 and 13 show the dependence of $\eta^{*}$ upon $K$ at various $\beta^{\prime}$. Table $\mathrm{V}$ lists interpolated values of $\eta^{*}$ vs $\beta^{\prime}$ and $K$ to allow construction of graph $\mathrm{s}^{17}$ similar to Figs. 11-13.

Thus, for any L-J $(12,6)$ system, the procedure for evaluating the scattering cross sections is as follows. From a given set of L-J parameters $(\sigma, \epsilon)$ one calculates $B$; knowing $\mu$ and $v$, one evaluates $A$ and $K$. For each angular momentum quantum number $l$ one calculates $\beta^{\prime}$, reads off the corresponding $\eta^{*}$ from the $Q-i-K$ curve and then calculates $\eta_{l}$. From the resulting table of phase shifts the computation of $d \sigma(\theta) / d \Omega[\equiv I(\theta)]$ is straightforward.

With the exception of the problem of the "bounded region" for cases when $K \leq 0.8$, Table $\mathrm{V}$ should suffice to enable estimation of the phase shifts without the

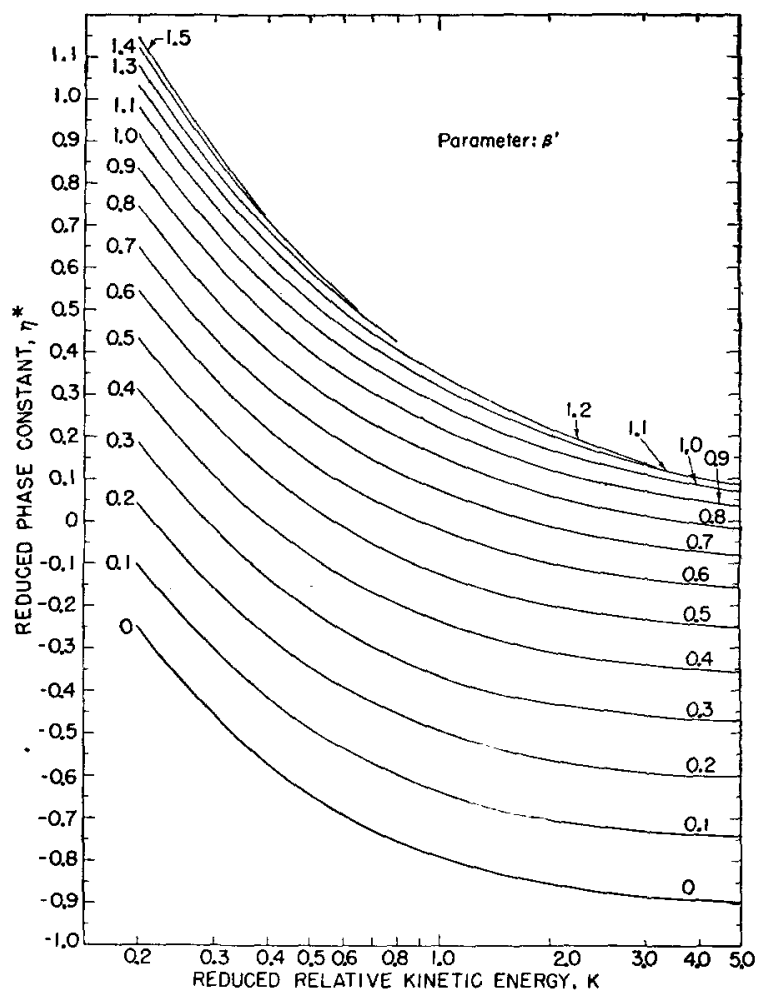

FIG. 12. Reduced phase constant vs reduced relative kinetic energy.

${ }^{17}$ In the "bounded region" of Figs. 11-13, delineated in Table $\mathrm{Vb}$, corresponding to the region of collision energies and angular momenta where orbiting or spiral scattering is possible, insufficient calculations of phase shifts were made to allow the precise location of the discontinuities in $\eta^{*}$ vs $\beta^{\prime}$ at each value of $K$. Thus, for any individual case it would be necessary to make a few direct calculations of $\eta$ in the neighborhood of the discontinuities.

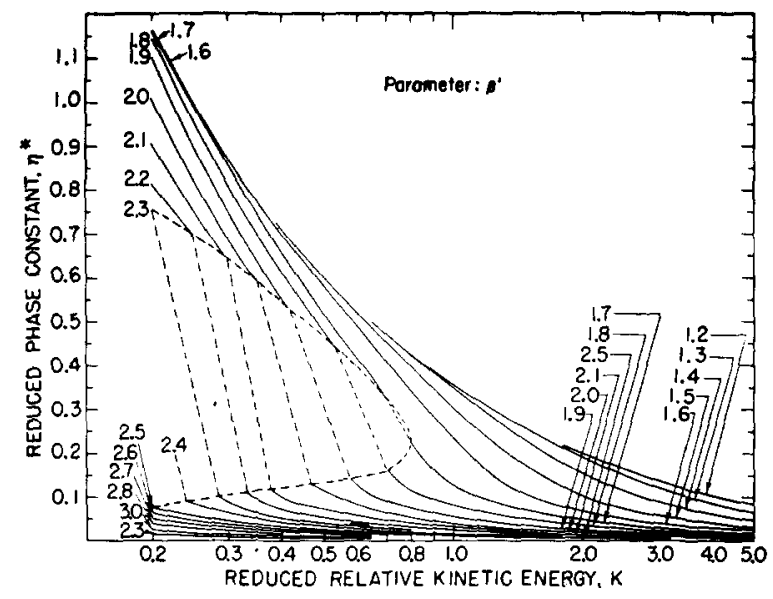

Frg. 13. Reduced phase constant vs reduced relative kinetic energy.

necessity of carrying out the numerical integration of the wave equation.

A preliminary study has been made of the errors introduced in the calculated cross sections due to (a) random errors [of the order of \pm 0.02 to 0.05 in $\eta_{l}$ ] and (b) omission of the small higher-order phases. Random errors do not significantly affect the general shape of the scattering curve, but rather introduce strong changes locally in a few regions. The "wavelength" of the undulations in $d \sigma / d \Omega$ is governed primarily by $1 / A$ (or $\lambda$ ) so that the positions of the maxima and minima are virtually unaffected. The error due to (b) is, however, more insidious; it affects the low angle scattering and thus may alter the total cross section $Q$ by a significant degree. In addition, it is obvious that the accuracy of the numerical integration decreases (and the computing time increases) with increasing $l$, so that the integration technique is unsuitable for the precise calculation of the very high order phases (e.g., $l>60$ ). For improved accuracy in $Q$ one should therefore use the Born approximation for the higher phase ${ }^{18}$ and include in the calculation all phase shifts greater than about $0.005 \mathrm{rad}$. Further work in this direction with particular attention to the problem of the total cross section and its wavelength dependence is in progress.

\section{ACKNOWLEDGMENTS}

The author wishes to express his appreciation to Dr. Kenneth Smith of the Argonne National Laboratory for his advice and assistance with the prototype of the computer program for the wave function. In addition, many valuable discussions with Dr. H. U. Hostettler and Dr. R. S. Berry of this Department are gratefully acknowledged. The assistance of Mrs. H. Schumacher in plotting graphs and arranging tables is also appreciated.

\footnotetext{
${ }^{18}$ It may be shown that the Born approximation for the higher order phases yields $\eta=(3 \pi / 8) B A^{4} / l^{5}$. For $\eta \leqq 0.5$ and $l \geqq 2 A$ this formula reproduces the directly calculated phases (cf. Table III) within \pm 0.01 .
} 


\section{APPENDIX}

TABLE Va. Reduced phase constants $\left(\eta^{*}\right)$ as a function of $K$ and $\beta^{\prime}$.

\begin{tabular}{|c|c|c|c|c|c|c|c|c|c|c|c|}
\hline$\beta^{\lambda}$ & 0.2 & 0.3 & 0.4 & 0.5 & 0.6 & 0.8 & 1.0 & 2.0 & 3.0 & 4.0 & 5.0 \\
\hline $\begin{array}{l}0 \\
0.1 \\
0.2 \\
0.3 \\
0.4 \\
0.5 \\
0.6 \\
0.7 \\
0.8 \\
0.9 \\
1.0 \\
1.1 \\
1.2 \\
1.3 \\
1.4 \\
1.5 \\
1.6 \\
1.7 \\
1.8 \\
1.9 \\
2.0 \\
2.1 \\
2.2 \\
2.3 \\
2.4 \\
2.5 \\
2.6 \\
2.7 \\
2.8 \\
3.0 \\
3.3\end{array}$ & $\begin{array}{r}-0.244 \\
-0.096 \\
0.050 \\
0.189 \\
0.314 \\
0.435 \\
0.548 \\
0.650 \\
0.748 \\
0.834 \\
0.912 \\
0.980 \\
1.038 \\
1.088 \\
1.128 \\
1.151 \\
1.166 \\
1.164 \\
1.145 \\
1.105 \\
1.013 \\
0.910 \\
0.817\end{array}$ & $\begin{array}{r}-0.454 \\
-0.298 \\
-0.152 \\
-0.016 \\
0.107 \\
0.230 \\
0.339 \\
0.433 \\
0.528 \\
0.615 \\
0.687 \\
0.746 \\
0.799 \\
0.834 \\
0.868 \\
0.885 \\
0.872 \\
0.859 \\
0.816 \\
0.755 \\
0.691 \\
0.645 \\
\\
0.090 \\
0.063 \\
0.045 \\
0.037 \\
0.030 \\
0.022 \\
0.013 \\
0.008\end{array}$ & $\begin{array}{r}-0.570 \\
-0.412 \\
-0.268 \\
-0.131 \\
-0.008 \\
0.112 \\
0.219 \\
0.317 \\
0.405 \\
0.487 \\
0.557 \\
0.610 \\
0.658 \\
0.690 \\
0.711 \\
0.713 \\
0.700 \\
0.663 \\
0.619 \\
0.553 \\
0.101 \\
0.072 \\
0.052 \\
0.041 \\
0.032 \\
0.027 \\
0.021 \\
0.016 \\
0.012 \\
0.007\end{array}$ & $\begin{array}{r}-0.642 \\
-0.487 \\
-0.341 \\
-0.206 \\
-0.080 \\
0.038 \\
0.140 \\
0.239 \\
0.325 \\
0.403 \\
0.470 \\
0.521 \\
0.563 \\
0.594 \\
0.609 \\
0.606 \\
0.586 \\
0.522 \\
0.481 \\
\\
0.117 \\
0.073 \\
0.053 \\
0.040 \\
0.032 \\
0.026 \\
0.020 \\
0.017 \\
\\
0.009 \\
0.006\end{array}$ & $\begin{array}{r}-0.692 \\
-0.534 \\
-0.390 \\
-0.258 \\
-0.131 \\
-0.015 \\
0.089 \\
0.184 \\
0.270 \\
0.347 \\
0.409 \\
0.458 \\
0.496 \\
0.523 \\
0.531 \\
0.521 \\
0.490 \\
0.418 \\
\\
0.128 \\
0.084 \\
0.058 \\
0.043 \\
0.032 \\
0.026 \\
0.021 \\
0.017 \\
0.012 \\
\\
0.008 \\
0.005\end{array}$ & $\begin{array}{r}-0.754 \\
-0.597 \\
-0.453 \\
-0.324 \\
-0.196 \\
-0.082 \\
0.024 \\
0.115 \\
0.200 \\
0.271 \\
0.330 \\
0.376 \\
0.406 \\
0.424 \\
0.424 \\
0.399 \\
0.344 \\
0.225 \\
0.115 \\
0.074 \\
0.053 \\
0.040 \\
0.030 \\
0.023 \\
0.018 \\
0.014 \\
0.011\end{array}$ & $\begin{array}{r}-0.790 \\
-0.636 \\
-0.492 \\
-0.366 \\
-0.237 \\
-0.125 \\
-0.020 \\
0.071 \\
0.153 \\
0.220 \\
0.276 \\
0.318 \\
0.348 \\
0.356 \\
0.350 \\
0.304 \\
0.237 \\
0.128 \\
0.082 \\
0.054 \\
0.038 \\
0.031 \\
0.024 \\
\\
0.013 \\
0.010\end{array}$ & $\begin{array}{r}-0.861 \\
-0.711 \\
-0.571 \\
-0.438 \\
-0.318 \\
-0.209 \\
-0.111 \\
-0.025 \\
0.048 \\
0.108 \\
0.153 \\
0.186 \\
0.199 \\
0.190 \\
0.154 \\
0.102 \\
0.068 \\
0.048 \\
0.033 \\
0.023 \\
0.019 \\
0.012\end{array}$ & $\begin{array}{r}-0.883 \\
-0.730 \\
-0.592 \\
-0.459 \\
-0.340 \\
-0.232 \\
-0.138 \\
-0.057 \\
0.012 \\
0.070 \\
0.110 \\
0.131 \\
0.137 \\
0.120 \\
0.088 \\
0.057 \\
0.039 \\
0.029 \\
0.021 \\
0.015 \\
0.011 \\
0.008\end{array}$ & $\begin{array}{r}-0.890 \\
-0.738 \\
-0.599 \\
-0.468 \\
-0.350 \\
-0.245 \\
-0.150 \\
-0.071 \\
-0.006 \\
0.050 \\
0.086 \\
0.102 \\
0.103 \\
0.086 \\
0.061 \\
0.040 \\
0.030 \\
0.020 \\
0.016 \\
0.010 \\
0.007\end{array}$ & $\begin{array}{r}-0.896 \\
-0.740 \\
-0.600 \\
-0.471 \\
-0.356 \\
-0.251 \\
-0.158 \\
-0.081 \\
-0.018 \\
0.036 \\
0.070 \\
0.087 \\
0.082 \\
0.063 \\
0.047 \\
0.030 \\
0.025 \\
0.017 \\
0.012 \\
0.007 \\
0.004\end{array}$ \\
\hline
\end{tabular}

TABLE Vb. Bounded region.

\begin{tabular}{|c|c|c|c|c|}
\hline \multirow[b]{2}{*}{$\beta^{\prime}$} & \multicolumn{2}{|c|}{ Lower } & \multicolumn{2}{|c|}{ Upper } \\
\hline & $K$ & $\eta^{*}$ & $K$ & $\eta^{*}$ \\
\hline 1.7 & 0.800 & 0.225 & 0.704 & 0.319 \\
\hline 1.8 & 0.707 & 0.160 & 0.538 & 0.440 \\
\hline 1.9 & 0.574 & 0.144 & 0.422 & 0.524 \\
\hline 2.0 & 0.468 & 0.131 & 0.353 & 0.590 \\
\hline 2.1 & 0.378 & 0.120 & 0.301 & 0.643 \\
\hline 2.2 & 0.333 & 0.110 & 0.249 & 0.700 \\
\hline 2.3 & 0.287 & 0.100 & 0.200 & 0.755 \\
\hline 2.4 & 0.240 & 0.090 & & \\
\hline
\end{tabular}

